\title{
STRIKING PHOTOSPHERIC ABUNDANCE ANOMALIES IN BLUE HORIZONTAL-BRANCH STARS IN GLOBULAR CLUSTER M13
}

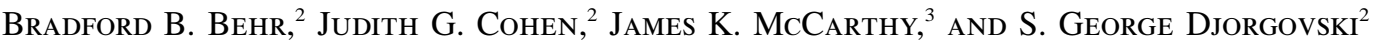 \\ Received 1999 March 11; accepted 1999 March 29; published 1999 April 22
}

\begin{abstract}
High-resolution optical spectra of 13 blue horizontal-branch stars in the globular cluster M13 show enormous deviations in element abundances from the expected cluster metallicity. In the hotter stars $\left(T_{\text {eff }}>12,000 \mathrm{~K}\right)$, helium is depleted by factors of 10-100 below solar, while iron is enhanced to 3 times the solar abundance, 2 orders of magnitude above the canonical metallicity of $[\mathrm{Fe} / \mathrm{H}] \simeq-1.5 \mathrm{dex}$ for this globular cluster. Nitrogen, phosphorus, and chromium exhibit even more pronounced enhancements, and other metals are also mildly overabundant, with the exception of magnesium, which stays very near the expected cluster metallicity. These photospheric anomalies are most likely due to diffusion-the gravitational settling of helium and the radiative levitation of the other elements - in the stable radiative atmospheres of these hot stars. The effects of these mechanisms may have some impact on the photometric morphology of the cluster's horizontal branch and on estimates of its age and distance.
\end{abstract}

Subject headings: globular clusters: general — globular clusters: individual (NGC 6205) — stars: abundances - stars: horizontal-branch

\section{INTRODUCTION}

A number of unresolved issues in post-main-sequence stellar evolution revolve around the nature of stars on the horizontal branch (HB), an evolutionary stage characterized by core helium burning and shell hydrogen burning. The HB stars in globular clusters are particularly appealing targets, since they are readily identified by their position in a cluster's colormagnitude diagram, and are assumed to be chemically homogeneous and coeval with the other stars in the cluster. Although intrinsically luminous, most cluster HB stars are also distant, with $V$ magnitudes of 14 or greater, so that detailed spectroscopic study is challenging. With the recent advent of 8-10 m-class telescopes and highly efficient spectrographs, however, the HBs of many of the nearer globular clusters are now accessible at high spectral resolution in reasonable exposure times. We have therefore undertaken a program to measure the chemical abundances and rotation rates of $\mathrm{HB}$ stars in M3, M13, M15, M92, and M68 via high-resolution echelle spectroscopy.

M13 (NGC 6205) is one of the closest and best-studied globulars, with $(m-M)=14.35$ mag (Peterson 1993) and a metallicity of $[\mathrm{Fe} / \mathrm{H}]=-1.51$ dex measured from red giant abundances (Kraft et al. 1992). Its blue horizontal branch (BHB) extends from the blue edge of the RR Lyrae gap to rather high temperatures (a "long blue tail") but is interrupted by one or more gaps, including a large one at $(U-V) \simeq-0.3$ mag. Some researchers have suggested that this gap separates two different populations of BHB stars (Ferraro et al. 1997b; Sosin et al. 1997). In order to test this hypothesis, we have observed stars on either side of the gap, to look for differences in composition and rotation. In this Letter, we describe the striking trends in helium and metal abundance that we observe

\footnotetext{
${ }^{1}$ Based in large part on observations obtained at the W. M. Keck Observatory, which is operated jointly by the California Institute of Technology and the University of California.

${ }^{2}$ Palomar Observatory, Mail Stop 105-24, California Institute of Technology, Pasadena, CA 91125.

${ }_{3}^{3}$ PixelVision, Inc., 4952 Warner Avenue, Suite 300, Huntington Beach, CA 92649.
}

along the $\mathrm{HB}$ of M13. The rotation results will be reported in a subsequent paper (Behr et al. 1999).

\section{OBSERVATIONS AND REDUCTION}

The spectra were collected using the HIRES spectrograph (Vogt et al. 1994) on the Keck I telescope, during four observing runs on 1998 June 27, August 20-21, August 26-27, and 1999 March 9-11. A 0"86 slit width yielded $R=45,000$ $\left(v=6.7 \mathrm{~km} \mathrm{~s}^{-1}\right)$ per 3 pixel resolution element. Spectral coverage ran from 3940 to $5440 \AA(m=90-66)$ for the June observations and from 3890 to $6280 \AA(m=91-57)$ for the August and March observations, with slight gaps above $5130 \AA$ where the free spectral range of the orders overfilled the detector. We limited frame exposure times to $1200 \mathrm{~s}$, in order to minimize susceptibility to cosmic-ray accumulation, and then co-added three frames per star. The signal-to-noise ratios were on the order of 50-90 per resolution element, permitting us to measure even weak lines in the spectra.

Nine of the 13 stars in our sample were selected from the Hubble Space Telescope (HST) Wide Field Planetary Camera 2 photometry of the center of M13 from Ferraro et al. (1997a), as reduced by M. Zoccali, E. Pancino, \& G. Piotto (1999, private communication). The program stars were selected to be as isolated as possible; the HST images show no apparent neighbors within $\simeq 5^{\prime \prime}$. The seeing during the HIRES observations was sufficiently good $\left(00^{\prime \prime} 8-1{ }^{\prime \prime} .0\right)$ to avoid any risk of spectral contamination. The $U-V$ colors from the HST study provided the $T_{\text {eff }}$ estimates for the subsequent abundance analysis. The other four M13 HB program stars were taken from the $v \sin i$ and $[\mathrm{O} / \mathrm{H}]$ survey of Peterson, Rood, \& Crocker (1995). They are all located in the cluster outskirts, where crowding is not so problematic. Positions, finding charts, photometry, and observational details for the target stars will be provided in a later paper (Behr 1999).

We used a suite of routines developed by McCarthy (1988) for the FIGARO data analysis package (Shortridge 1993) to reduce the HIRES echellegrams to one-dimensional spectra. Frames were bias-subtracted, flat-fielded against exposures of HIRES's internal quartz incandescent lamps (thereby removing much of the blaze profile from each order), cleaned of cosmic- 
ray hits, and co-added. A thorium-argon arc lamp provided wavelength calibration. Sky background was negligible, and one-dimensional spectra were extracted via simple pixel summation. A 10th-order polynomial fit to line-free continuum regions completed the normalization of the spectrum to unity.

\section{ANALYSIS}

The resulting spectra show many tens to over 200 metal absorption lines each. Line broadening from stellar rotation is evident in many stars, but even in the most extreme cases, the line profiles were close to Gaussian, so line equivalent widths $\left(W_{\lambda}\right)$ were measured by least-squares fitting of Gaussian profiles to the data. Equivalent widths as small as $10 \mathrm{~m} \AA$ were measured reliably, and errors in $W_{\lambda}$ (estimated from the fit $\chi^{2}$ ) were typically $5 \mathrm{~mA}$ or less.

Observed lines were matched to the atomic line lists of Kurucz \& Bell (1995). (Several observed lines in the hotter stars could not be identified, and a more comprehensive future analysis will attempt to do so.) Those lines that were identified provided a consistent $v_{r}$ solution for each of the stars, placing all of them well within the canonical heliocentric $v_{r}=-246.6 \mathrm{~km} \mathrm{~s}^{-1}$ of M13.

We make the simplifying assumption that all the program stars lie on or near the zero-age horizontal-branch (ZAHB) track computed by Dorman, Rood, \& O'Connell (1993), so that surface gravity would be determined by our choice of temperature. For the hotter and potentially "overluminous" HB stars (discussed below), this assumption may overestimate $\log g$ by as much as $\simeq 0.7$ dex (Moehler 1999, Fig. 4), but this will have only a modest impact on computed abundances for the species observed. Effective temperature was derived for the nine $H S T$ stars by matching dereddened $U-V$ color indices to computed ATLAS9 colors, with errors in $T_{\text {eff }}$ based on the photometric errors. For the four non-HST stars, we accepted the published $T_{\text {eff }}$ values of Peterson et al. (1995), although we note that these are based on photographic $B-V$ photometry and are thus suspect. Strömgren photometry of M13 will refine the $T_{\text {eff }}$ for these stars in a later reanalysis. We assign conservative error bars of $\pm 500 \mathrm{~K}$ to the photographic temperatures.

For the chemical abundance analyses, we use the LINFOR/ LINFIT line formation analysis package (developed at Kiel, based on earlier codes by Baschek, Holweger, \& Traving 1966, with subsequent modifications by M. Lemke), along with model atmospheres computed by ATLAS9 (Kurucz 1997). Our spectra are sufficiently uncrowded that we can simply compute abundances from equivalent widths instead of performing a full spectral synthesis fit. Only lines attributed to a single chemical species were considered; potentially blended lines are ignored in this analysis. Microturbulent velocity $\xi$ was chosen such that the abundance derived for a single species (Fe II) was invariant with $W_{\lambda}$. We assumed a cluster metallicity of $[\mathrm{Fe} / \mathrm{H}]=-1.5$ dex in computing the model atmospheres, and although many of the stars turn out to be considerably more metal-rich than this (see below), adjustments to the atmospheric input were found to have only modest effects $(<0.2 \mathrm{dex})$ on the abundances of individual elements. In Table 1, we list the final photospheric parameters used for each of the target stars as well as the heliocentric radial velocities.

\section{RESULTS}

In Figure 1, abundance determinations for key chemical species are plotted as a function of stellar $T_{\text {eff }}$. Note that the bottom three panels have a different vertical scale than the top six. The
TABLE 1

Photospheric Parameters for Program Stars

\begin{tabular}{|c|c|c|c|c|}
\hline Star & $\begin{array}{l}T_{\text {eff }} \\
(\mathrm{K})\end{array}$ & $\log g$ & $\begin{array}{c}\xi \\
\left(\mathrm{km} \mathrm{s}^{-1}\right)\end{array}$ & $\begin{array}{c}\text { Heliocentric } \\
v_{r} \\
\left(\mathrm{~km} \mathrm{~s}^{-1}\right)\end{array}$ \\
\hline IV-83 ... & $8960 \pm 500$ & $3.37 \pm 0.40$ & $3 \pm 1$ & -253.4 \\
\hline SA 113 & $11580 \pm 500$ & $3.90 \pm 0.40$ & $3 \pm 1$ & -241.9 \\
\hline SA 404 & $10940 \pm 500$ & $3.78 \pm 0.40$ & $1 \pm 1$ & -250.8 \\
\hline $\mathrm{J} 11 \ldots \ldots$ & $7780 \pm 500$ & $3.10 \pm 0.40$ & $3 \pm 1$ & -246.7 \\
\hline WF2-3035 & $8470 \pm 110$ & $3.26 \pm 0.40$ & $2 \pm 1$ & -253.7 \\
\hline WF2-2541 & $16970 \pm 210$ & $4.72 \pm 0.40$ & $1 \pm 1$ & -257.9 \\
\hline WF2-2692 & $16860 \pm 200$ & $4.71 \pm 0.40$ & $2 \pm 1$ & -236.2 \\
\hline WF4-3085 & $15300 \pm 200$ & $4.50 \pm 0.40$ & $0+1$ & -255.9 \\
\hline WF2-820 . & $14520 \pm 240$ & $4.39 \pm 0.40$ & $0+1$ & -240.3 \\
\hline WF3-548 & $16820 \pm 190$ & $4.70 \pm 0.40$ & $2 \pm 1$ & -235.7 \\
\hline WF4-3485 & $13760 \pm 200$ & $4.27 \pm 0.40$ & $0+1$ & -247.3 \\
\hline WF3-1718 & $11480 \pm 140$ & $3.88 \pm 0.40$ & $1 \pm 1$ & -244.4 \\
\hline WF2-3123 & $19220 \pm 310$ & $4.98 \pm 0.40$ & $1 \pm 1$ & -238.5 \\
\hline
\end{tabular}

values $[\mathrm{X} / \mathrm{H}]$ represent logarithmic offsets from the solar values of Anders \& Grevesse (1991). Whenever possible, we used the abundance computed for the dominant ionization stage of each element, to minimize the possibility of non-LTE effects. The error bars incorporate the scatter among multiple lines of the same species plus the uncertainties in $T_{\text {eff }}, \log g, \xi, W_{\lambda}$ for each line, and $[\mathrm{Fe} / \mathrm{H}]$ of the input atmosphere. Even with the conservative error bars in $T_{\text {eff }}( \pm 500 \mathrm{~K}$ in the cooler stars) and $\log g( \pm 0.4 \mathrm{dex})$, individual element abundances are uncertain by 0.3 dex or less, with the sole exception of the $\mathrm{Ca}$ I lines of star IV-83.

The abundances of helium, iron, and magnesium provide the most striking contrast in behavior. The He abundance first appears at the expected solar $\mathrm{He} / \mathrm{H}$ ratio at $T_{\text {eff }} \simeq 11,000 \mathrm{~K}$ but then drops by a factor of more than 100 as $T_{\text {eff }}$ increases to 19,000 K. Similarly, iron is present at (or slightly below) the $[\mathrm{Fe} / \mathrm{H}]=-1.51 \mathrm{dex}$ expected for this metal-poor cluster but then rises to Population I abundances for the stars hotter than $\simeq 12,000 \mathrm{~K}$. Magnesium, on the other hand, appears consistently at almost exactly the canonical cluster metallicity, with no discernable change with $T_{\text {eff }}$.

Other metals exhibit similar enhancements in the hotter stars. The $\mathrm{Ti}$ abundance rises by approximately a factor of 30 from 8000 to $15,000 \mathrm{~K}$, although the trend is not as clear-cut as with iron. Silicon and calcium are also modestly enhanced to $[\mathrm{X} / \mathrm{H}] \simeq-0.5 \mathrm{dex}$ among some of the hotter stars. The most pronounced overabundances are seen in phosphorus, which appears at $[\mathrm{P} / \mathrm{H}] \simeq 1.5 \mathrm{dex}$ in six stars, and chromium, which climbs past solar metallicity to reach a remarkable $[\mathrm{Cr} / \mathrm{H}]=$ 3.10 dex, an enhancement of more than a factor of $10^{4}$ over the metallicity of M13, albeit in only one star. Each of these values is based on several separate spectral lines, in close agreement with each other, so we are confident that they are not due to random errors or line misidentification.

The CNO elements, particularly nitrogen, also show enhancements, although most of these abundances are based on only a single line per species and are therefore suspect. N II appears in four of the hot stars, at $[\mathrm{N} / \mathrm{H}]$ ranging from 1.6 to 3.5 dex. Nitrogen enhancement from dredge-up of fusionprocessed material is expected in evolved stars, but not to this extent, so if these values are accurate, some other mechanism must be at work. A single oxygen line appears at a more reasonable $[\mathrm{O} / \mathrm{H}]=1.0 \mathrm{dex}$, and carbon is solar or slightly subsolar in three of the stars. 


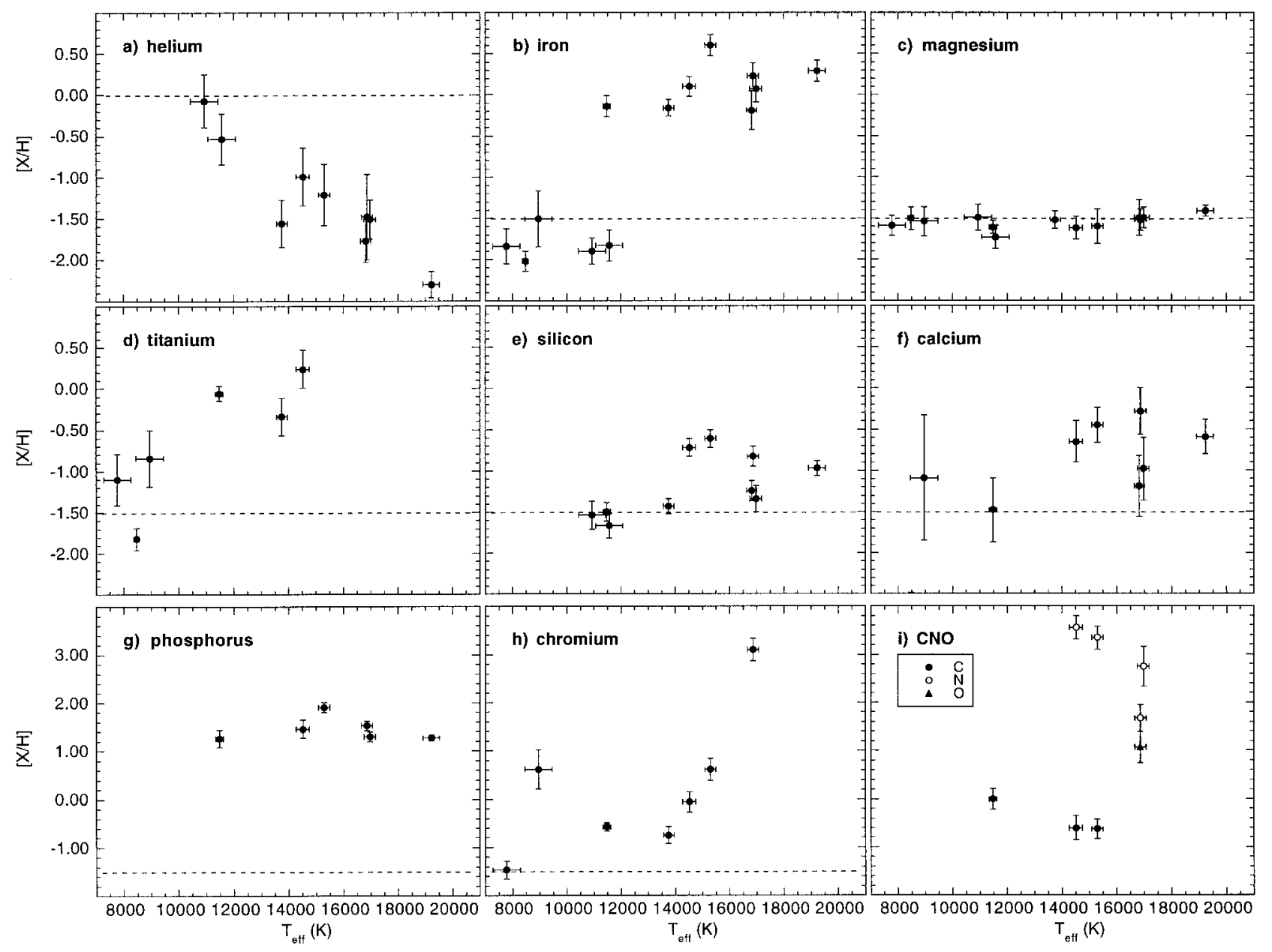

Fig. 1.- $\mathrm{He}, \mathrm{Fe}, \mathrm{Mg}, \mathrm{Ti}, \mathrm{Si}, \mathrm{Ca}, \mathrm{P}, \mathrm{Cr}$, and $\mathrm{CNO}$ abundances over a range of $T_{\text {eff, }}$ as log offsets from the solar abundances. The dotted line represents the expected value of $[\mathrm{X} / \mathrm{H}]$ for stars in M13 (ignoring dredge-up of fusion-processed or $\alpha$-enhanced material): -1.51 dex from solar for the metals and the solar ratio for helium. Note that the vertical scale is different in the bottom three panels.

\section{DISCUSSION}

An underabundance of helium on the BHB has been observed in several previous instances (Baschek 1975; Heber 1987; Glaspey et al. 1989; among others) and in fact appears to be typical for stars of this type. Michaud, Vauclair, \& Vauclair (1983, hereafter MVV), building on the original suggestion by Greenstein, Truran, \& Cameron (1967), explain the underabundances as a result of the gravitational settling of helium, which can take place if the outer atmosphere of the star is sufficiently stable. Our current results are significant in that they demonstrate a distinct trend in $[\mathrm{He} / \mathrm{H}]$ with $T_{\text {eff }}$ and $\log g$ along the HB, including cooler, lower gravity stars with roughly solar helium abundances, such that the magnitude of helium diffusion can be traced over a range of conditions. Although MVV do predict greater helium depletion in their hotter, higher gravity BHB models, the actual abundance pattern is also likely to depend on the stellar rotation rate. As pointed out in both MVV and Glaspey et al. (1989), diffusion can easily be stymied by turbulence, mass loss, and meridional circulation produced by stellar rotation. Many of the BHB stars in M13 are comparatively fast rotators (Peterson et al. 1995), reaching $v \sin i \simeq 40 \mathrm{~km} \mathrm{~s}^{-1}$, while the 10 stars in our study with helium abundances all exhibit extremely narrow lines, suggesting $v \sin i<6 \mathrm{~km} \mathrm{~s}^{-1}$ in most cases. A more comprehensive assessment of the stellar rotations, and their potential effects on diffusion processes, will be presented in another paper (Behr et al. 1999).

The MVV calculations indicate that helium depletion should be accompanied by photospheric enhancement of metals, since the same stable atmosphere that permits gravitational settling also permits the levitation of species with large radiative cross sections. Overabundances of factors of $10^{3}-10^{4}$ from a star's initial composition could be supported by radiation pressure, although, as Glaspey et al. hasten to point out, this is a "necessary but not sufficient condition" for actual abundance anomalies to appear, given the possibility of turbulent mixing or the radiation-driven escape of metals from the star. MVV make some initial assessment of the magnitudes of these variations, but future models will have to explain more fully why some elements $(\mathrm{N}, \mathrm{P}$, and $\mathrm{Cr}$ ) are enhanced so much more strongly than others $(\mathrm{Fe}, \mathrm{C}, \mathrm{Ti}, \mathrm{Ca}$, and $\mathrm{Si}$ ), while a few $(\mathrm{Mg})$ are apparently immune to diffusion mechanisms. None of the recent diffusion work reported in the literature treats the specific case of the BHB, so a detailed comparison of our results with current theory will have to wait for improved models.

Our results for iron and magnesium closely parallel those of Glaspey et al., who studied two stars in globular cluster NGC 6752 , one at $10,000 \mathrm{~K}$ and the other at $16,000 \mathrm{~K}$. The hotter star displays an $\mathrm{Fe}$ enhancement that is 50 times above the cluster mean, but the cooler star has the same $[\mathrm{Fe} / \mathrm{H}]$ as 
the cluster, while the $\mathrm{Mg}$ abundances are near the cluster mean in both cases. They find significantly lower amounts of silicon and phosphorus than we do, but the rough agreement in the helium and iron anomalies between these two BHB stars in NGC 6752 and our larger sample in M13 suggests that these diffusion mechanisms are not peculiar to M13.

In addition to furthering our understanding of diffusion mechanisms, these atmospheric abundance variations offer potential ramifications for the photometric morphology of globular clusters. A great deal of recent attention has focused on the presence of gaps in the color distribution of stars on the HBs of M13 and several other clusters (Ferraro et al. 1997b; Sosin et al. 1997; Buonanno et al. 1986). The origin of such gaps is not yet understood and presents a challenge for theories of HB evolution. A prominent gap in M13's BHB, located at $T_{\text {eff }} \simeq 11,000 \mathrm{~K}$ and labeled "G1" in Ferraro et al. (1997b), seems to coincide with the onset of our diffusion anomalies. We will explore this possible connection in later publications.

Additionally, there is the issue of "overluminous" regions of the BHB in several clusters including M13 (Moehler 1999; Grundahl, VandenBerg, \& Anderson 1998; Grundahl et al. 1999). While cooler BHB stars are in good agreement with theoretical ZAHB tracks, those in the range $11,000 \mathrm{~K} \leq$ $T_{\text {eff }} \leq 20,000 \mathrm{~K}$ are found to be significantly brighter (or, equivalently, at lower $\log g$ ) than expected. Again, this is the temperature range where diffusion effects start to alter the atmospheric composition significantly, possibly also modifying the atmospheric structure. The abundances that we observe should prove useful in evaluating the potential role of diffusion-driven metal enhancement in explaining this phenomenon.

Lastly, the observed helium diffusion may have some impact on estimates of globular cluster ages. Helium diffusion in mainsequence models can alter evolutionary timescales by $10 \%$ or more (VandenBerg, Bolte, \& Stetson 1996), and although the atmospheric structures of HB stars and main-sequence stars are quite different, the magnitude of helium diffusion seen at different $T_{\text {eff }}$ and $\log g$ on the HB may offer some insights into the degree of diffusion expected in the main-sequence case. More importantly, the helium fraction can influence the luminosity of the ZAHB (Proffitt 1997), which will affect age determinations based on the $\Delta V$ between the turnoff and the $\mathrm{HB}$, as well as distance estimates using the observed magnitudes of HB stars. Further observational and theoretical work will be necessary to determine what relationship (if any) exists between the onset of these diffusion-driven abundance anomalies and other characteristics of the HB, such as its luminosity, stellar rotation, and gaps in its color distribution, and whether diffusion significantly affects estimates of globular cluster ages.

These observations would not have been feasible without the HIRES spectrograph and the Keck I telescope. We are indebted to Jerry Nelson, Gerry Smith, Steve Vogt, and many others for making such marvelous machines, to the W. M. Keck Foundation for making it happen, and to a bevy of Keck observing assistants for making them work. Patrick Côté graciously provided assistance with many of the HIRES observations. Thanks also go to Manuela Zoccali, Elena Pancino, and Giampaolo Piotto for their reduction of the HST photometry and to Michael Lemke for introducing us to the LINFOR package and installing it locally. S. G. D. was supported, in part, by the Bressler Foundation. This research has made use of the SIMBAD database, operated at CDS, Strasbourg, France.

\section{REFERENCES}

Anders, E., \& Grevesse, N. 1991, in Solar Interior and Atmosphere, ed. A. N. Cox, W. C. Livingston, \& M. S. Matthews (Tucson: Univ. Arizona Press), 1227

Baschek, B. 1975, in Problems in Stellar Atmospheres and Envelopes, ed. B. Baschek, W. H. Kegel, \& G. Traving (New York: Springer), 101

Baschek, B., Holweger, H., \& Traving, G. 1966, AAHam, 8, 26

Behr, B. B. 1999, in preparation

Behr, B. B., et al. 1999, in preparation

Buonanno, R., Caloi, V., Castellani, V., Corsi, C., Fusi Pecci, F., \& Gratton, R. 1986, A\&AS, 66, 79

Dorman, B., Rood, R. T., \& O'Connell, W. O. 1993, ApJ, 419, 596

Ferraro, F. R., Paltrinieri, B., Fusi Pecci, F., Cacciari, C., Dorman, B., \& Rood, R. T. 1997a, ApJ, 484, L145

Ferraro, F. R., Paltrinieri, B., Fusi Pecci, F., Dorman, B., \& Rood, R. T. 1997b, ApJ, 500, 311

Glaspey, J. W., Michaud, G., Moffat, A. F. J., \& Demers, S. 1989, ApJ, 339, 926

Greenstein, G. S., Truran, J. W., \& Cameron, A. G. W. 1967, Nature, 213, 871

Grundahl, F., Catelan, M., Landsman, W. B., Stetson, P. B., \& Andersen, M. I. 1999, ApJ, submitted (astro-ph/9903120)

Grundahl, F., VandenBerg, D. A., \& Anderson, M. I. 1998, ApJ, 500, L179
Heber, U. 1987, Mitt. Astron. Ges., 70, 79

Kraft, R. P., Sneden, C., Langer, G. E., \& Prosser, C. F. 1992, AJ, 104, 645 Kurucz, R. L., \& Bell, B. 1995, Atomic Line Data, Kurucz CD-ROM 23 (Cambridge: SAO)

Kurucz, R. L. 1997, in The Third Conf. on Faint Blue Stars, ed. A. G. D. Philip, J. W. Leibert, \& R. A. Saffer (Schenectady: Davis), 33

McCarthy, J. K. 1988, Ph.D. thesis, Caltech

Michaud, G., Vauclair, G., \& Vauclair, S. 1983, ApJ, 267, 256 (MVV)

Moehler, S. 1999, Rev. Mod. Astron., in press (astro-ph/9812147)

Peterson, C. 1993, in ASP Conf. Ser. 50, Structure and Dynamics of Globular Clusters, ed. S. G. Djorgovski \& G. Meylan (San Francisco: ASP), 337

Peterson, R. C., Rood, R. T., \& Crocker, D. A. 1995, ApJ, 453, 214

Proffitt, C. R. 1997, in IAU Symp. 189, Fundamental Stellar Properties: The Interaction between Observation and Theory, ed. T. R. Bedding, A. J. Booth, \& J. Davis (Boston: Kluwer), 355

Shortridge, K. 1993, in ASP Conf. Ser. 52, Astronomical Data Analysis Software and Systems II, ed. R. J. Hanisch, R. J. V. Brissenden, \& J. Barnes (San Francisco: ASP), 219

Sosin, C., et al. 1997, ApJ, 480, L35

VandenBerg, D. A., Bolte, M., \& Stetson, P. B. 1996, ARA\&A, 34, 461

Vogt, S. E., et al. 1994, Proc. SPIE, 2198, 362 